Supporting Information for:

\title{
Photocatalytic Conversion of Nitrobenzene to Aniline through Sequential Proton-Coupled One-Electron Transfers from a Cadmium Sulfide Quantum Dot
}

\author{
Stephen C. Jensen, Stephanie Bettis Homan, and Emily A. Weiss* \\ Department of Chemistry, Northwestern University, 2145 Sheridan Rd., Evanston, IL 60208- \\ 3113 \\ *corresponding author. Email: e-weiss@northwestern.edu
}

\section{Supporting Information Table of Contents:}

\section{Supporting Figures}

Figure S1. Expanded Figure 1C with ${ }^{1} \mathrm{H}-\mathrm{NMR}$ of MPA regions .............................. 3

Figure S2. Sample 1H-NMR plot used to calculate EQE from Figure 2 ......................S4

Figure S3. Sample GC spectrum before and after illumination ............................. 5

Figure S4. UV-Vis and photoemission spectra of QDs under $54 \mathrm{hr}$. of illumination ............S7

Figure S5. NB, NSB, PHA, and AN with and without MPA before and after CdS QDs .........S9

Figure S6. DOSY spectrum for MPA QDs and Nitrobenzene .............................. 10

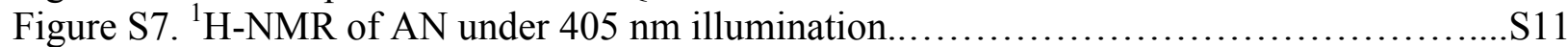

Figure S8. ${ }^{1} \mathrm{H}-\mathrm{NMR}$ of AN with the addition of MPA and triton B ............................ 12

Figure S9. Photoluminescence quenching of CdS QDs in presence of NB.....................S12

Figure S10. Kinetic Traces of Excited-State Decay of CdS QDs with NB and MPA...........S13

\section{Supporting Tables}

Table S1. NB, PHA, AN, MPA, Disulfide concentrations calculated from Figure S1 ..........S3

Table S2. Rate constants from global fit of GC kinetics ......................................S7

Table S3. Time Constants of Excited-State Decay of CdS QDs with NB and MPA............S14 
Preparation of MPA-capped CdS QDs. In a 3-neck round-bottomed flask, we heat $0.256 \mathrm{~g}$ CdO (Sigma-Aldrich \#244783), $13.7 \mathrm{~mL}$ octadecene (ODE, Sigma-Aldrich, 90\%), and $6.3 \mathrm{~mL}$ oleic acid (OA, Sigma-Aldrich, $90 \%$ ) to $250{ }^{\circ} \mathrm{C}$ under a flow of nitrogen. We ensure that all of the $\mathrm{CdO}$ is converted to cadmium oleate by checking that the solution is clear, and we remove 4 $\mathrm{mL}$ of the solution and store the rest for future use. We combine $4 \mathrm{~mL}$ of cadmium oleate with 6 $\mathrm{mL} \mathrm{ODE}$ and heat the solution under nitrogen to $260^{\circ} \mathrm{C}$. To initiate the QD growth, we inject 2 $\mathrm{mL} 0.10 \mathrm{M}$ sulfur in ODE and reduce the temperature to $220^{\circ} \mathrm{C}$. We allow the oleate-capped QDs to nucleate and grow for 1.5 minutes and verify that they have a first absorption peak at $\sim 407 \mathrm{~nm}$. The QDs are purified by splitting the QD mixture into $4 \times 15-\mathrm{mL}$ centrifuge tubes, adding 3:1 acetone:QDs, and centrifuging the tubes at $3500 \mathrm{rpm}$ for 5 minutes. The supernatant is yellow and the pellet of QDs is bright yellow. We discard the supernatant, redisperse the pellet in 1-2 mL hexanes, add 3:1 (v:v) methanol:QDs, centrifuge at $3500 \mathrm{rpm}$ for 5 minutes, discard the supernatant, and redisperse in chloroform. We allow the QDs to sit for at least 24 hours before any additional treatment.

We next perform a ligand exchange to replace oleate ligands with MPA ligands. We perform the ligand exchange on small volumes of QDs, as needed, since the MPA-coated QDs are sensitive to oxygen and precipitate from solution over time. We concentrate $\sim 50 \mathrm{nmol}$ of the QDs so that they occupy $0.25 \mathrm{~mL}$ or less and add $0.5 \mathrm{~mL}$ MPA (Sigma-Aldrich, $>99 \%$ ), $6 \mathrm{~mL}$ methanol, and $7 \mathrm{~mL}$ Triton B (Sigma-Aldrich, $40 \mathrm{wt}$ \% in methanol) to deprotonate the thiol and facilitate the ligand exchange. We stir the solution for at least $2.5 \mathrm{hr}$, transfer it to $4 \times 15 \mathrm{~mL}$ centrifuge tubes, add $5 \mathrm{~mL}$ ethyl ether (Sigma-Aldrich, 99.0\%) and $5 \mathrm{~mL}$ ethyl acetate (SigmaAldrich, 99.8\%) to each tube, and centrifuge at $3500 \mathrm{rpm}$ for 5 minutes. We discard the supernatant, redisperse in $4 \mathrm{~mL}$ methanol, and then mix with $5 \mathrm{~mL}$ ethyl ether and $5 \mathrm{~mL}$ ethyl acetate. We centrifuge the tubes again at $3500 \mathrm{rpm}$ for 5 minutes, remove the supernatant, and redisperse in methanol. After washing, there is a trace amount of Triton B left in solution $(<0.3$ $\mathrm{mM}$ ), but, as detected by ${ }^{1} \mathrm{H}-\mathrm{NMR}$, the Triton $\mathrm{B}$ is not reactive even under illumination of the sample. The solution is purged with nitrogen or argon for 5 minutes and stored in a nitrogen box for 24 hours before use. 

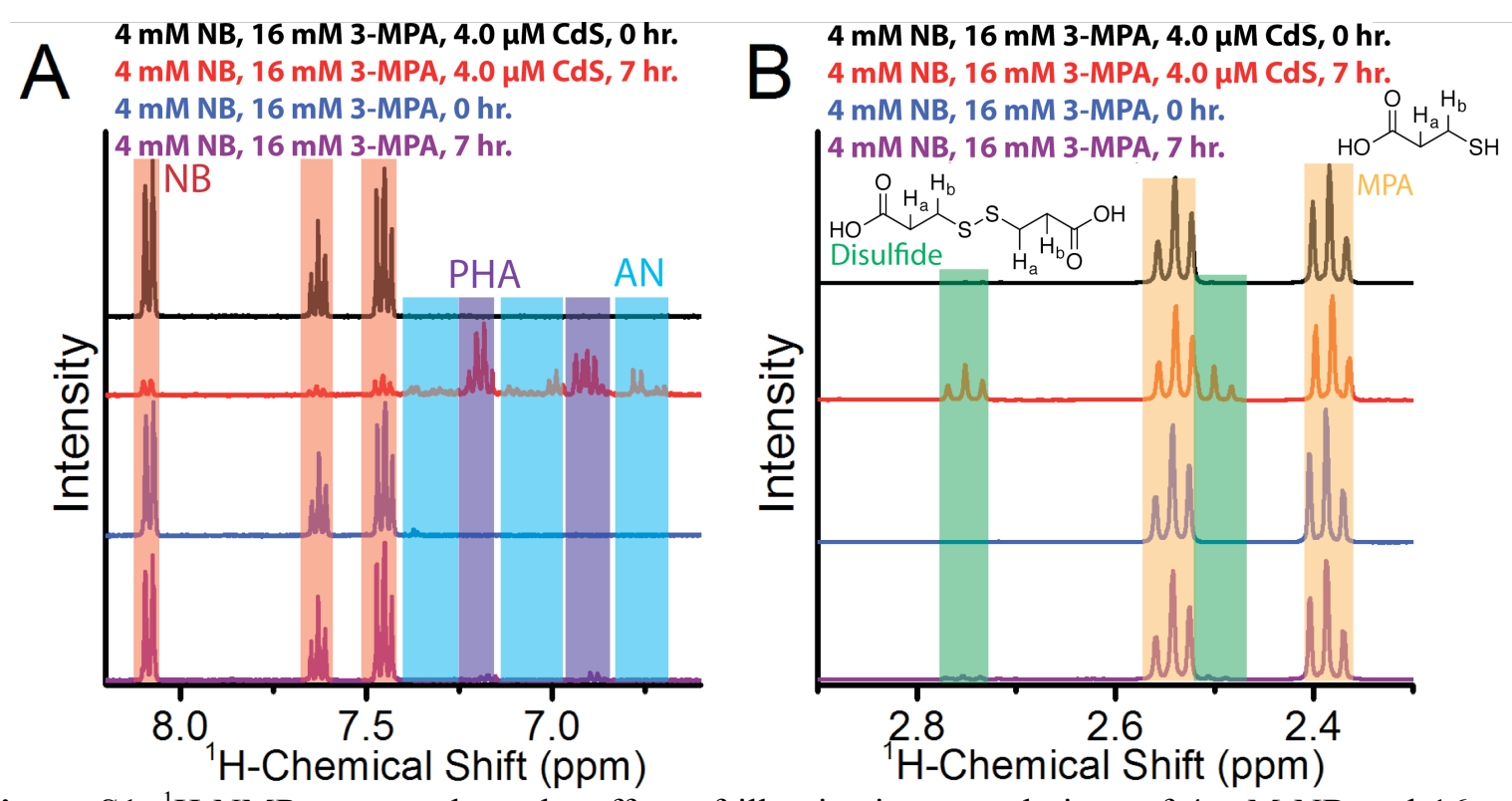

Figure S1. ${ }^{1} \mathrm{H}-\mathrm{NMR}$ spectra show the effect of illumination on solutions of $4 \mathrm{mM} \mathrm{NB}$ and $16 \mathrm{mM}$ MPA in the presence and absence of $4 \mu \mathrm{M}$ CdS QDs in 80:20 $\mathrm{CD}_{3} \mathrm{OD}: \mathrm{D}_{2} \mathrm{O}$. (A) Spectra taken from Figure $1 \mathrm{C}$ of the main text show peaks that correspond to phenyl ring protons of NB (red bars), PHA (purple bars), and AN (blue bars). Solutions with QDs (black trace) and without QDs (blue trace) are illuminated for 7 hours and spectra for both samples are taken after (red and purple traces, respectively). (B) ${ }^{1} \mathrm{H}-\mathrm{NMR}$ spectra of the protons in MPA with (black) and without (blue) CdS QDs before illumination and after $7 \mathrm{hr}$ illumination (red and purple, respectively) show the formation of the disulfide, indicated by peaks in the green regions.

MPA is oxidized to 3,3-Dithiodipropionic acid as a result of the photo-reduction. ${ }^{1} \mathrm{H}$ NMR results reveal that, prior to illumination, an 80:20 CD ${ }_{3} \mathrm{OD}: \mathrm{D}_{2} \mathrm{O}$ solution containing $4 \mathrm{mM}$ $\mathrm{NB}, 16 \mathrm{mM}$ MPA, and $4.0 \mu \mathrm{M}$ CdS QDs show two triplet peaks near 2.55 and $2.38 \mathrm{ppm}$ that are characteristic of MPA (Figure S1B black trace). Upon illumination, the peak intensity drops and a new set of triplet peaks form, which are shifted $0.2 \mathrm{ppm}$ downfield and characteristic of 3,3dithiodipropionic acid (red trace). No disulfide peaks are produced without the addition of CdS QDs (purple trace). Table S1 lists the results of quantitative analysis of these spectra.

Table S1. NB, PHA, AN, MPA, and Disulfide concentrations calculated from Figure S1.

$\begin{array}{ccc} & \text { no QD, 7 hr (mM) } & \text { w/ QD, 7 hr (mM) } \\ \text { NB } & 3.9 & 0.7 \\ \text { PHA } & 0.1 & 2.3 \\ \text { AN } & 0.0 & 1.1 \\ \text { MPA } & 14.6 & 10.9 \\ \text { Disulfide } & 0.2 & 2.0\end{array}$




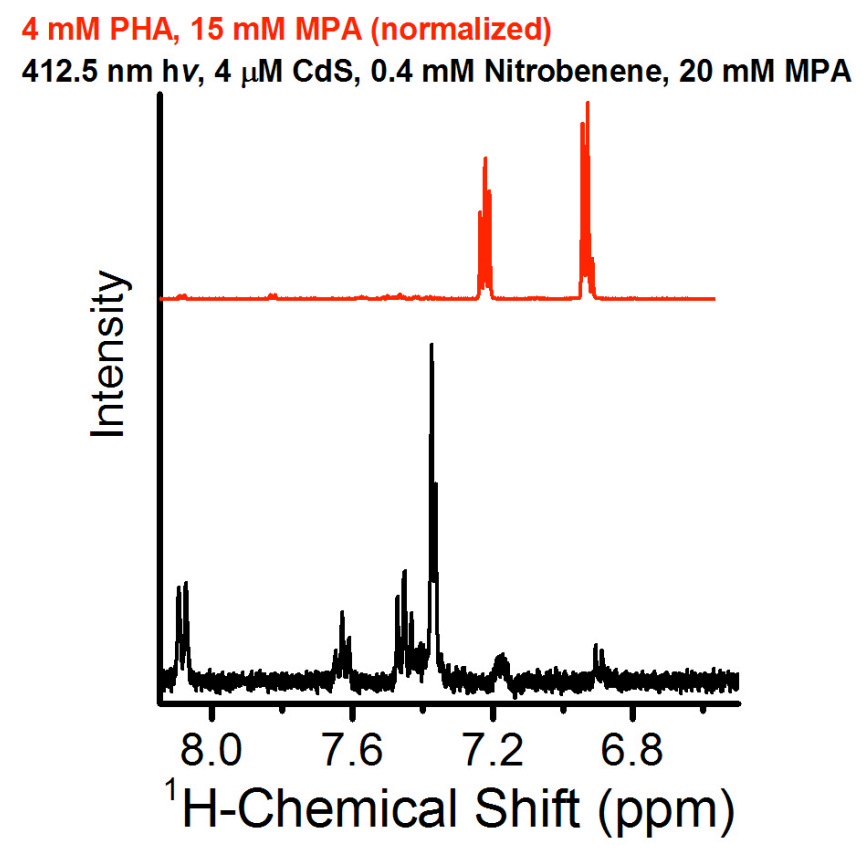

Figure S2. ${ }^{1} \mathrm{H}-\mathrm{NMR}$ spectrum for the sample illuminated with $412.5 \mathrm{~nm}$ light for 4 minutes that was used to calculate an EQE for that wavelength in the Action Spectrum in Figure 2. We measure the concentration of PHA from two characteristic peaks at 7.2 and $6.9 \mathrm{ppm}$. A scaled spectrum of $4 \mathrm{mM}$ PHA and $15 \mathrm{mM}$ MPA is shown for reference.

External Quantum Efficiency Calculation. A solution of $0.4 \mathrm{mM} \mathrm{NB}, 20 \mathrm{mM}$ MPA in 80:20 $\mathrm{D}_{2} \mathrm{O}: \mathrm{CD}_{3} \mathrm{OD}$ is illuminated with $\sim 5 \mathrm{~mW}$ at several wavelengths and we quantify in NMR the concentration of PHA that we produce after illuminating for 4 minutes. As shown in the black trace of Figure S2, nitrobenzene peaks at 8.1 and $7.6 \mathrm{ppm}$ are still present, but new peaks at 7.2 and $6.9 \mathrm{ppm}$ occur which align with a control sample with PHA and MPA. The tall peak at 7.5 corresponds to Triton B which comes from trace amounts left from the QD ligand exchange. We divide the concentration of PHA produced by the number of incident photons to get an external quantum yield for the reaction, $\mathrm{EQE}_{\mathrm{PHA}}$, and then convert to the external quantum yield per single electron transfer, $\mathrm{EQE}_{\text {electron, using eq. } 1 .}$

$$
E Q E_{\text {electron }}=\left(E Q E_{P H A}\right)^{1 / 4}
$$




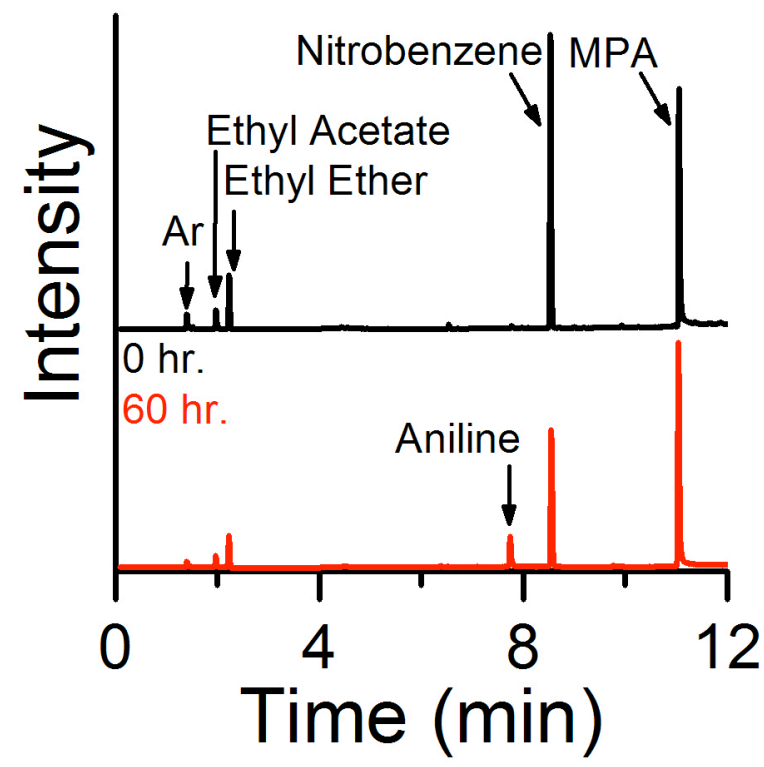

Figure S3. Characteristic GC spectra for $4 \mathrm{mM} \mathrm{NB}, 20 \mathrm{mM}$ MPA, $40 \mathrm{nM}$ CdS QDs before (black) and after (red) $60 \mathrm{hr}$ illumination with a $7 \mathrm{~mW} 405 \mathrm{~nm}$ laser while stirring. The full integrated analysis for all of these plots is detailed in Figures 1A,B of the main text.

PHA and AN Product Quantification from GC-MS. We quantify the concentration of PHA and AN by first running a concentration curve for each molecule in the GC-MS. Both PHA and $\mathrm{AN}$ desorb exclusively as $\mathrm{AN}$ from the GC-column, however the integrated signal intensity for PHA at a particular concentration is $\sim 10 \%$ of what would be expected for AN at the same concentration. Since we know from NMR that the concentration of aromatic molecules is conserved during the photo-reduction of NB, and that the only products are PHA and AN, the smaller apparent product yield is instead due to the smaller cracking fraction of PHA compared to aniline. The concentrations of $\mathrm{AN}$ and PHA can thus be determined by calculating the concentration of products from the amount of remaining NB (eq S2), setting the signal of apparent products equal to a sum of both PHA and AN calibration curves (eq S3), and solving for the concentration of PHA and AN. "Noise" observed in Figure 1A and 1B is due to sampling inconsistencies in the autosampler in the GC-MS.

$$
\begin{aligned}
& {[\text { Products }]_{t}=[\mathrm{NB}]_{0}-[\mathrm{NB}]_{t}} \\
& \text { MS ProductSignal }{ }_{t}=f\left([\mathrm{NB}]_{t}\right)-g\left([\mathrm{NB}]_{t}\right)
\end{aligned}
$$

Modeling of GC-MS Time Traces. The rate equation for 3 sequential, reversible reactions is as follows: 


$$
\begin{aligned}
& N B \underset{k_{N S B-N B}}{\stackrel{k_{N B N B}}{\rightleftharpoons}} N S B \underset{k_{P H A-N S B}}{\stackrel{k_{N S-P H A}}{\rightleftharpoons}} P H A \underset{k_{A N-P H A}}{\stackrel{k_{P H A-A N}}{\rightleftharpoons}} A N \\
& \partial N B / d t=-k_{N B-N S B} N B+k_{N S B-N B} N S B \\
& \partial N S B / d t=k_{N B-N S B} N B-\left(k_{N S B-N B}+k_{N S B-P H A}\right) N S B+k_{N S B-P H A} P H A \\
& \partial P H A / d t=k_{N S B-P H A} N B-\left(k_{P H A-N S B}+k_{P H A-A N}\right) P H A+k_{A N-P H A} A N \\
& \partial A N / d t=k_{P H A-A N} P H A-k_{A N-P H A} A N \\
& N S B[0]=0, \quad P H A[0]=0, \quad A N[0]=0
\end{aligned}
$$

The rates $\mathrm{k}_{\mathrm{NB}-\mathrm{NSB}}, \mathrm{k}_{\mathrm{NSB}-\mathrm{PHA}}, \mathrm{k}_{\mathrm{PHA}-\mathrm{AN}}$ designate the forward reactions while $\mathrm{k}_{\mathrm{NSB}-\mathrm{NB}}$, $\mathrm{k}_{\mathrm{PHA}-\mathrm{NSB}}$, $\mathrm{k}_{\mathrm{AN}-\mathrm{PHA}}$ are the back reactions. The starting concentration for all products are set to 0 , while $[\mathrm{NB}]$ is allowed to float. We utilize Mathematica 10 for symbolically deriving all differential equations. Mathematica was unable to compute the symbolic solution to the set of differential equations with all six rates, so we first solve the system for the forward reactions only to derive approximate solutions for the kinetic rates and the initial NB concentration.

$$
\begin{aligned}
& {[N B]_{t}=[N B]_{0} e^{-t k_{N B-N S B}}} \\
& {[N S B]_{t}=\frac{-[N B]_{0} k_{N B-N S B} e^{-t k_{N B-N S B}-t k_{N S B-P H A}}\left(-e^{t k_{N B-N S B}}+e^{t k_{N S B-P H A}}\right)}{k_{N B-N S B}-k_{N S B-P H A}}} \\
& {[P H A]_{t}=\left([ N B ] _ { 0 } k _ { N B - N S B } k _ { N S B - P H A } e ^ { - t k _ { N B - N S B } - t k _ { N S B - P H A } - t k _ { P H A } - A N } \left(k_{N B-N S B} e^{t k_{N B-N S B}+t k_{N S B-P H A}}\right.\right.}
\end{aligned}
$$

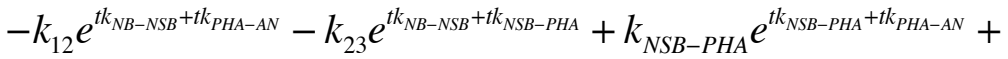

$$
\begin{aligned}
& \left.\left.k_{P H A-A N} e^{t k_{N B-N S B}+t k_{P H A-A N}}-k_{P H A-A N} e^{t k_{N S B}-P H A}+t k_{P H A-A N}\right)\right) / \\
& \left(\left(k_{N B-N S B}-k_{N S B-P H A}\right)\left(k_{N B-N S B}-k_{N S B-P H A}\right)\left(k_{N S B-P H A}-k_{P H A-A N}\right)\right) \\
& {[A N]_{t}=\left([ N B ] _ { 0 } e ^ { - t k _ { N B - N S B } - t k _ { N S B - P H A } - t k _ { P A A } - A N } \left(-k_{N B-N S B}^{2} k_{N S B-P H A} e^{t k_{N B-N S B}+t k_{N S B}-P H A}+\right.\right.} \\
& k_{N B-N S B}^{2} k_{N S B-P H A} e^{t k_{N B-N S B}+t k_{N S B-P H A}+t k_{P H A-A N}}-k_{N B-N S B}^{2} k_{N S B-P H A} e^{t k_{N B-N S B}+t k_{N S B}-P H A}- \\
& k_{N B-N S B}^{2} k_{N S B-P H A} e^{t k_{N B-N S B}+t k_{N S B-P H A}+t k_{P H A}-A N}+k_{N B-N S B}^{2} k_{P H A-A N} e^{t k_{N B-N S B}+t k_{P H A-A N}}+
\end{aligned}
$$

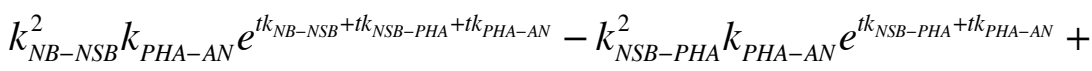

$$
\begin{aligned}
& k_{N S B-P H A}^{2} k_{P H A-A N} e^{t k_{N B-N S B}+t k_{N S B}-P H A}+t k_{P H A-A N}-k_{N B-N S B} k_{P H A-A N}^{2} e^{t k_{N B-N S B}+1 k_{P H A}-A V}+
\end{aligned}
$$

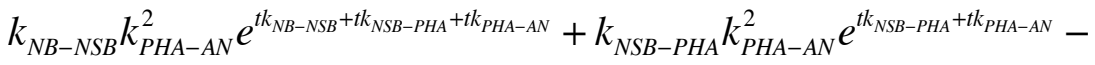

$$
\begin{aligned}
& \left.\left.k_{N S B-P H A} k_{P H A-A N}^{2} e^{t k_{N B-N S B}+k_{N S B}-P H A}+k_{P H A-A N}\right)\right) /\left(\left(k_{N B-N S B}-k_{N S B-P H A}\right) \times\right. \\
& \left.\left(k_{N B-N S B}-k_{N S B-P H A}\right)\left(k_{N S B-P H A}-k_{P H A-A N}\right)\right)
\end{aligned}
$$

We performed a global fitting analysis with the GC data for NB, PHA, and AN using Origin 9.0 for the values of $[\mathrm{NB}]_{0}, \mathrm{k}_{\mathrm{NB}-\mathrm{NSB}}, \mathrm{k}_{\mathrm{NSB}-\mathrm{PHA}}, \mathrm{k}_{\mathrm{PHA}-\mathrm{AN}}$. The fit did not converge when we performed a similar analysis for only 2 sequential reactions instead of 3 . Once we derived the forward rates and the initial concentration, we symbolically solved the set of differential 
equations for 1 irreversible followed by 2 reversible reactions and also in the case where the irreversible reaction was in the middle (symbolic equations not shown due to space considerations). The rates for both are given in Table S2.

Table S2. Best fit to GC data for reverse rates in kinetic model.

\begin{tabular}{|l|l|l|l|l|l|l|}
\hline Kinetic Model & $\boldsymbol{k}_{\text {NB-NSB }}$ & $\boldsymbol{k}_{\text {NSB-PHA }}$ & $\boldsymbol{k}_{P H A-A N}$ & $\boldsymbol{k}_{N S B-N B}$ & $\boldsymbol{k}_{P H A-N S B}$ & $\boldsymbol{k}_{A N-P H A}$ \\
\hline$N B \rightarrow N S B \leftrightarrow P H A \leftrightarrow A N$ & 0.0312 & 0.0107 & 0.0552 & --- & 0.002 & 0.009 \\
\hline$N B \leftrightarrow N S B \rightarrow P H A \leftrightarrow A N$ & 0.0312 & 0.0107 & 0.0552 & 0.000 & --- & 0.012 \\
\hline
\end{tabular}

${ }^{a}$ Forward rates are kept constant.

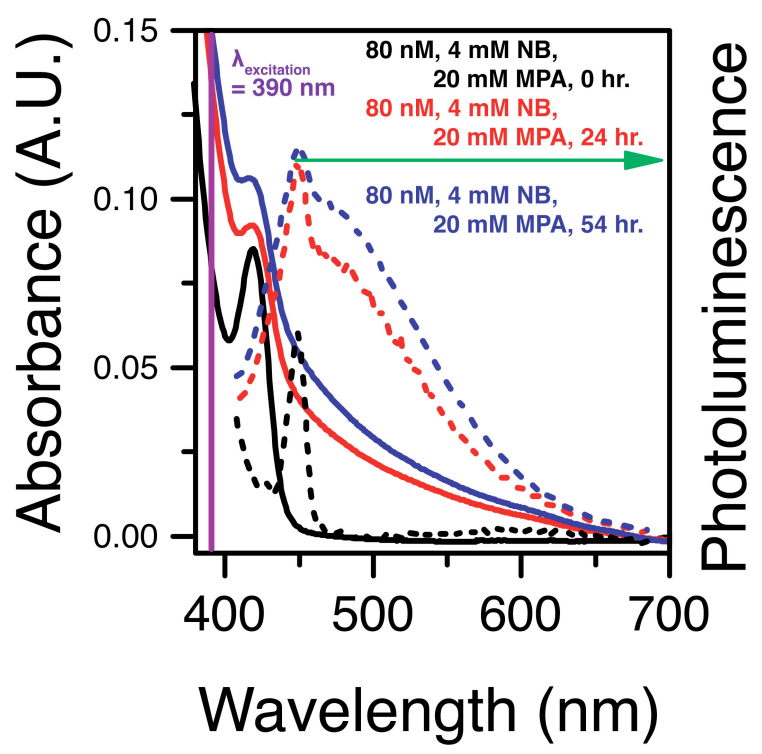

Figure S4. The absorption and emission spectra of $80 \mathrm{nM}$ MPA CdS QDs before and after $54 \mathrm{hr}$. illumination. A $80 \mathrm{nM} 3-\mathrm{MPA}$ CdS QD sample is prepared with $4 \mathrm{mM} \mathrm{NB}$ and $20 \mathrm{mM}$ MPA in $3 \mathrm{~mL}$ of a 20:80 solution of $\mathrm{CH}_{3} \mathrm{OH}: \mathrm{H}_{2} \mathrm{O}$. The solution is degassed by argon for 5 minutes, followed by a $\mathrm{UV}-\mathrm{Vis}$ (black solid line) and a photoemission spectra (black dotted line). The solution is illuminated for $24 \mathrm{hr}$. with a $7 \mathrm{~mW} 405 \mathrm{~nm}$ laser diode while stirring, followed by a UV-Vis and PL spectra (red solid and dotted lines, respectively) and then further illuminated for an additional $30 \mathrm{~h}$ before the final absorption and PL spectra are taken (blue solid and dotted lines). For all emission experiments, the QDs are illuminated at $390 \mathrm{~nm}$. 
Calculating the Percentage of molecules protonated at a given $\mathbf{p H}$. We calculate the protonation percent for a molecule using eq. S6:

$$
p H=p K a+\log \left[\frac{[A]^{-}}{1-[A]^{-}}\right]
$$

Once we determine the fraction that is unprotonated $[\mathrm{A}]^{-}$, we just subtract that fraction from 1 to find the fraction of molecules that are protonated at a given $\mathrm{pH},[\mathrm{HA}]$.

Calculating the Photon Flux. We measure an incident photon flux of $4.6 \times 10^{17}$ photons $\mathrm{cm}^{-2}$ $\mathrm{s}^{-1}$ and know from the absorption spectra that at $4 \mathrm{nM}$ and $0.8 \mathrm{~mm}$ path length, we only adsorb $1.1 \%$ of the incoming photons. This means we are creating $5.06 \times 10^{15}$ excitons $\mathrm{cm}^{-2} \mathrm{~s}^{-1}$. Assuming a rapid mixing speed, which we have due to a magnetic stirring bar, and a total number of QDs $\left(3.6 \times 10^{12}\right.$ per $1.5 \mathrm{~mL}$ vial, which is dictated by the concentration), the number of excitons created per second is given by eq S7.

$$
\frac{\text { Number of Excitons }}{\text { Number of Quantum Dots } \times \mathrm{sec}}=140.6 \text { photons } \mathrm{QD}^{-1} \mathrm{~s}^{-1}=7.11 \mathrm{~ms} \text { photon }^{-1} \quad \text { (eq. S7) }
$$



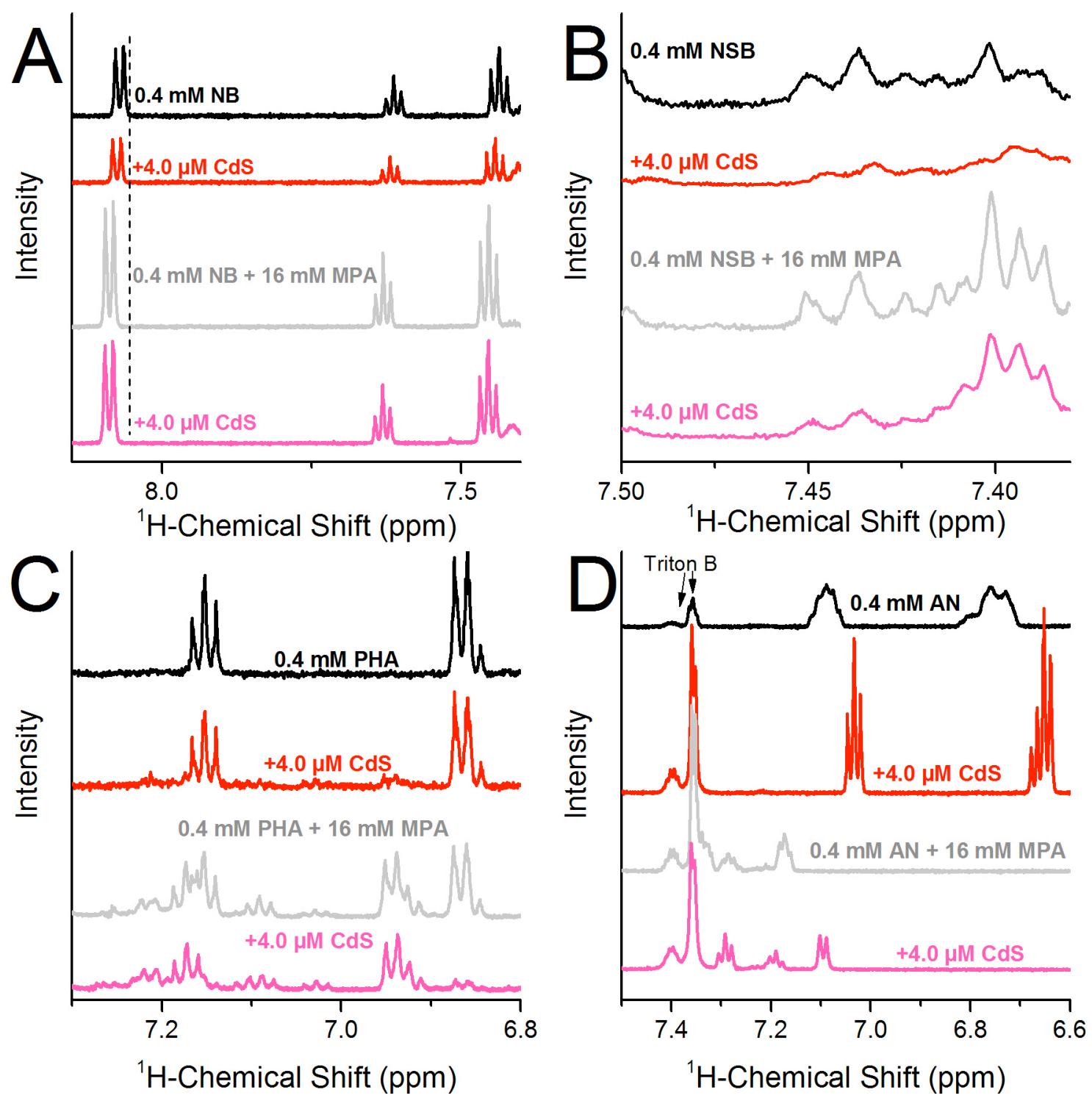

Figure S5. ${ }^{1} \mathrm{H}-\mathrm{NMR}$ spectra of NB (A), NSB (B), PHA (C), or AN (D) with (black) or without (gray) $16 \mathrm{mM}$ MPA. Samples are first analyzed and then mixed with $4 \mu \mathrm{M}$ 3-MPA CdS QDs and allowed to settle for $1 \mathrm{hr}$ before analyzing again (red and pink traces). The samples of NSB were identical to the others but the concentration of NSB and QDs was increased by $50 \%$ to permit detection in the NMR. All sample intensities are referenced to a $1 \mathrm{mM}$ ethylene glycol internal standard. The dotted line in (A) is for reference to show that the addition of MPA to NB causes an $0.01 \mathrm{ppm}$ downfield shift, which is due to formation of $[\mathrm{NB} \mathrm{H}]^{+}$. 


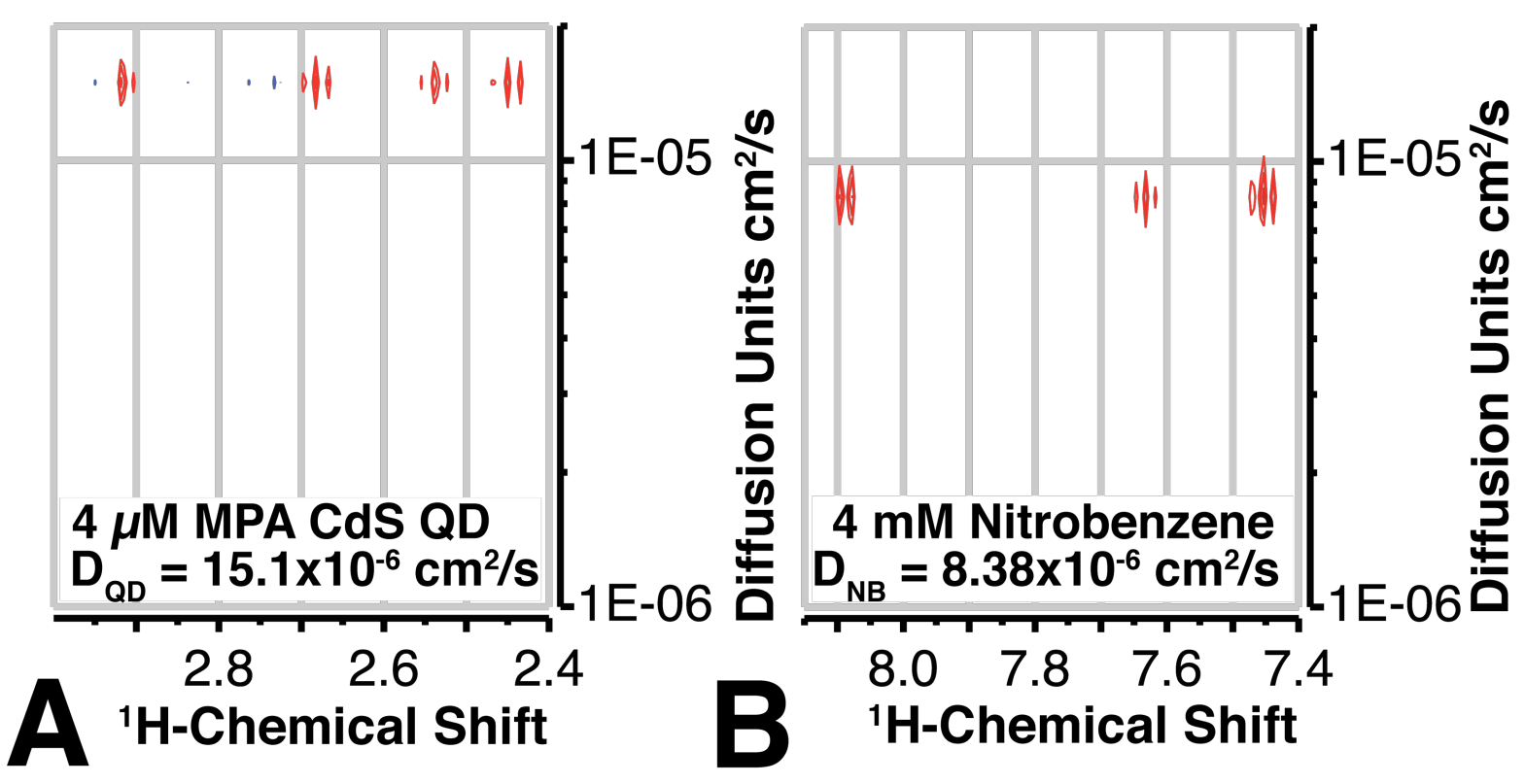

Figure S6. DOSY spectrum for MPA QDs and Nitrobenzene. (A) $4 \mu \mathrm{M}$ CdS in $100 \% \mathrm{D}_{3} \mathrm{OD}$. Four peaks are characteristic of MPA and the disulfide. No additional peaks with a slower diffusion time are present which leads us to conclude that the MPA species we detect are of the free molecule and not bound to the QD. (B) $4 \mathrm{mM}$ Nitrobenzene and $4 \mathrm{mM}$ MPA in 20:80 $\mathrm{D}_{3} \mathrm{OD}: \mathrm{H}_{2} \mathrm{O}$.

Calculating the Diffusion-Limited Time Constant. The diffusion-limited time constant, $\mathrm{k}_{0}$, is given by eq $\mathrm{S} 8$,

$$
k_{0}=\frac{4 \pi N_{A}}{1000}\left(R_{Q D}+R_{N B}\right)\left(D_{Q D}+D_{N B}\right)
$$

where $\mathrm{N}_{\mathrm{A}}$ is Avogadro's Number, $\mathrm{R}_{\mathrm{QD}}$ is the radius of the quantum dot, $\mathrm{R}_{\mathrm{NB}}$ is the radius of nitrobenzene, $\mathrm{D}_{\mathrm{QD}}$ is the diffusion constant of the quantum dot, and $\mathrm{D}_{\mathrm{NB}}$ is the diffusion constant of nitrobenzene. We utilize $3.72 \mathrm{~nm}$ dia. QDs, estimate the diameter of nitrobenzene at $0.56 \mathrm{~nm}$, and measure a diffusion constant for nitrobenzene in $80: 20$ methanol:water as $8.38 \times 10^{-6} \mathrm{~cm}^{2} / \mathrm{s}$ (Figure S6B). We attempted to measure the diffusion constant of the MPA QDs, but due to the short chain length of MPA, and broadening that occurs due to the molecules being bound to the QD, we only detect the diffusion constant of free MPA in solution $\left(15.1 \times 10^{-6} \mathrm{~cm}^{2} / \mathrm{s}\right.$, Figure $\mathrm{S} 6 \mathrm{~A})$. Instead, we utilize the diffusion rate for oleate capped $\mathrm{PbS}$ QDs in $\mathrm{CD}_{2} \mathrm{Cl}_{2}\left(2.13 \times 10^{-6}\right.$ $\left.\mathrm{cm}^{2} / \mathrm{s}\right) .{ }^{1}$ The longer chain length of the oleate permits detecting it in ${ }^{1} \mathrm{H}-\mathrm{NMR}$ even when bound to the QD. We calculate a resulting diffusion-limited time constant of $1.70 \times 10^{10} \mathrm{M}^{-1} \mathrm{~s}^{-1}$. We can convert this into a rate constant by multiplying by the concentration of quencher in our system, 
which in the case for nitrobenzene is initially $2 \mathrm{mM}$. This results in a diffusion-limited time constant of $29.4 \mathrm{~ns}$.

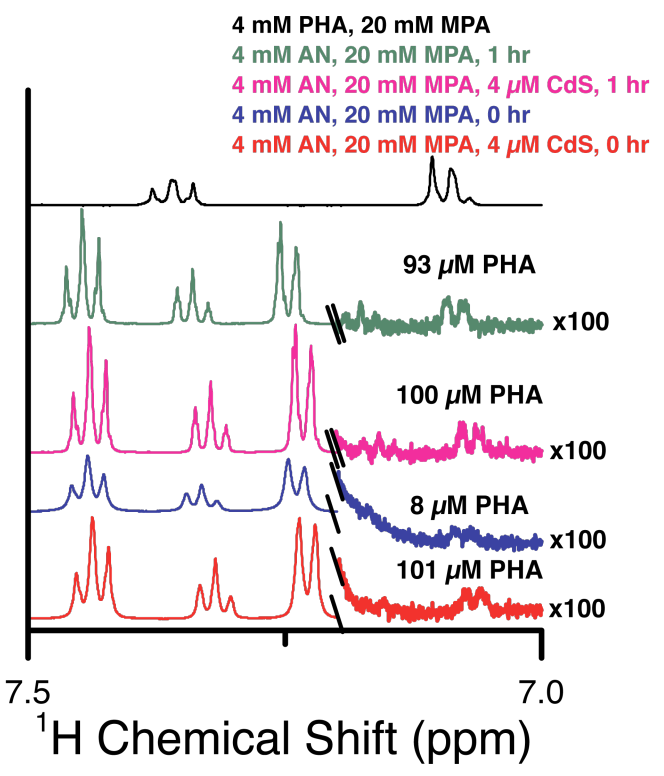

Figure S7. ${ }^{1} \mathrm{H}-\mathrm{NMR}$ spectra of AN with (red) and without (blue) CdS QDs before and after illumination (pink and green, respectively). Two samples of $4 \mathrm{mM} A \mathrm{~N}$ and $20 \mathrm{mM}$ MPA are prepared in a 20:80 solution of $\mathrm{CD}_{3} \mathrm{OD}: \mathrm{D}_{2} \mathrm{O}$ with and without $4 \mu \mathrm{M} \mathrm{CdS}$ QDs. The samples are analyzed in the NMR and then degassed with argon for 4 minutes each before being illuminated with $15 \mathrm{~mW} 405 \mathrm{~nm}$ illumination for $1 \mathrm{hr}$ while stirring in a $1.5 \mathrm{~mL}$ GC vial. The samples are then transferred back into an NMR tube for post illumination analysis. A sample of $4 \mathrm{mM}$ PHA and $20 \mathrm{mM}$ MPA is used as a control (black). Estimated concentrations of PHA for each sample is given above the PHA peak and the hash marks denote the demarcation between the normal and the $100 \times$ zoomed in section of the graph on the right. 


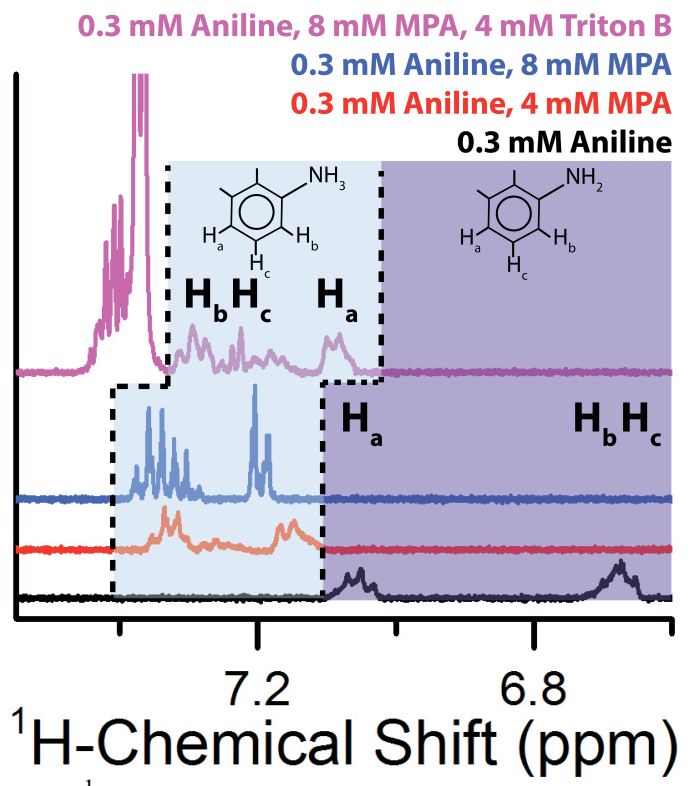

Figure S8. Aromatic regions of the ${ }^{1} \mathrm{H}-\mathrm{NMR}$ spectra of $0.3 \mathrm{mM}$ AN (black) after the sequential addition of $4 \mathrm{mM}$ MPA (red), $8 \mathrm{mM}$ MPA (black), and $4 \mathrm{mM}$ Triton B (purple). The peaks corresponding to the aromatic protons of AN (highlighted in purple) shift downfield and broadens when the molecule is protonated to the anilinium ion (highlighted in blue) in the presence of $8 \mathrm{mM} \mathrm{MPA}$ at $\mathrm{pH} 4.6$. The addition of Triton B shifts the proton signals upfield.

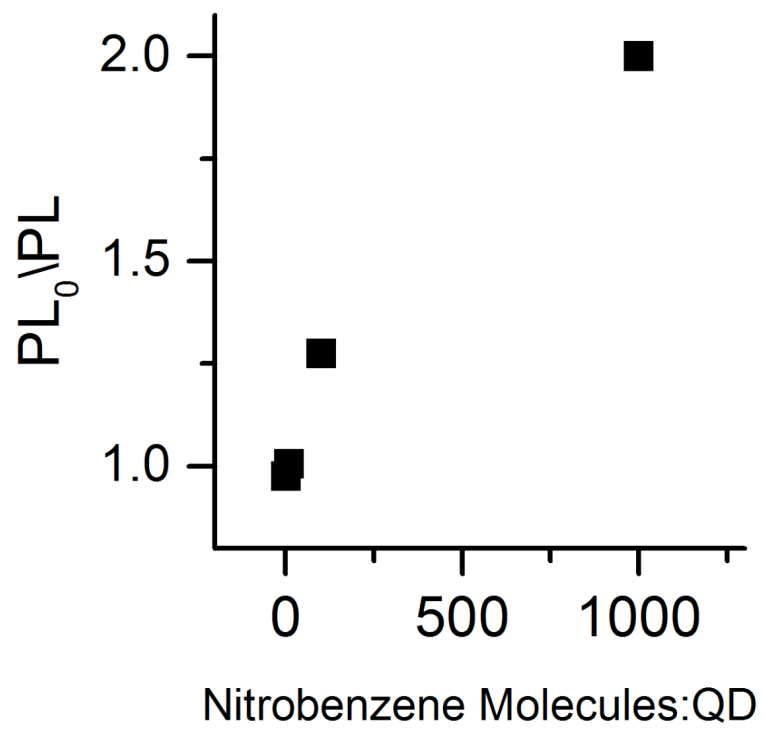

Figure S9. Plot of $\mathrm{PL}_{0} / \mathrm{PL}$ - the ratio of the original integrated intensity of the PL of $1 \mu \mathrm{M}$ CdS QDs to its intensity after addition of NB - in the absence of added MPA, such that any PL quenching occurs through electron transfer to NB. 
Deconvoluting the Electron and Hole Intraband Dynamics. The ground state bleach of CdS QDs reflects the electron dynamics exclusively, and so when we fit the decay kinetics at $1250 \mathrm{~nm}$ in Figure 3C, we only assign components to the hole if these components are not also present in the ground state bleach dynamics. We further support this method of deconvolution by examining the dynamics at $950 \mathrm{~nm}$, which includes contributions from the electron and hole. The dynamics we assign to the hole at $1250 \mathrm{~nm}$ appear at $950 \mathrm{~nm}$ but not in the bleach at $420 \mathrm{~nm}$ (see Figure S10 and Table S3, below). With these assignments, $950 \mathrm{~nm}$ is $\sim 60 \%$ hole, $40 \%$ electron; $420 \mathrm{~nm}$ is $100 \%$ electron; and $1250 \mathrm{~nm}$ is $87 \%$ hole and $13 \%$ electron. Analysis at these three wavelengths together substantiates our assignments of hole dynamics.

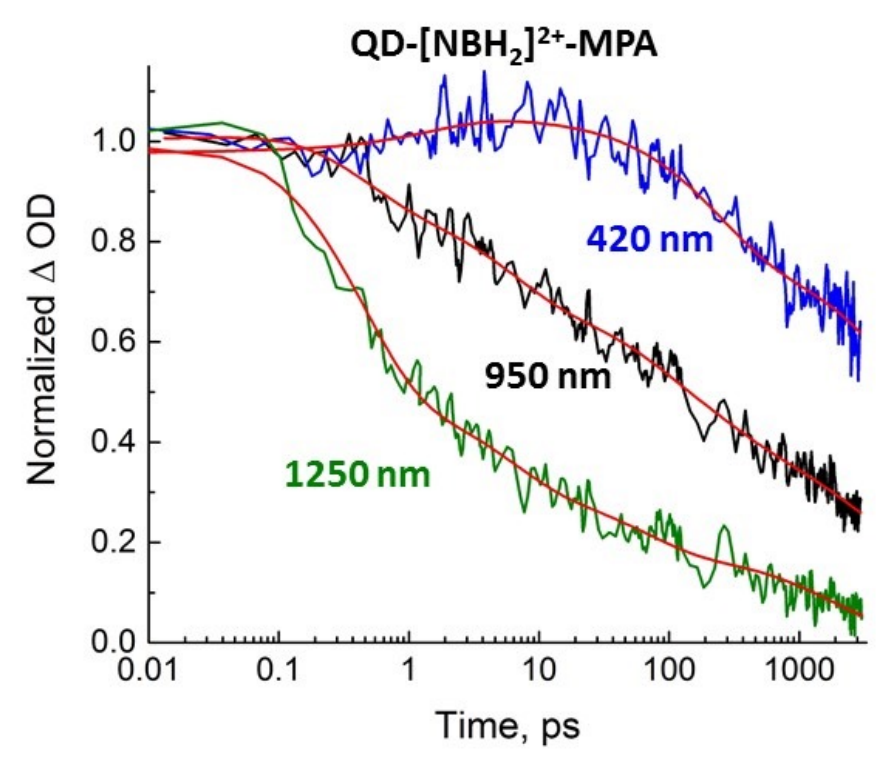

Figure S10. Normalized kinetic traces extracted from the TA spectra of $2 \mu \mathrm{M}$ CdS QDs with 1000 eq of added NB and 1000 eq of added 3-MPA, at three probe wavelengths: $420 \mathrm{~nm}$ (blue), $950 \mathrm{~nm}$ (black), and $1250 \mathrm{~nm}$ (green), after excitation at $390 \mathrm{~nm}$. The signal at $420 \mathrm{~nm}$, the ground state bleach, which is inverted here for ease of comparison, is dominated by the dynamics of the excitonic electron. The signal at $1250 \mathrm{~nm}$ is dominated by the dynamics of the excitonic hole. The signal at $950 \mathrm{~nm}$ is a mixture. The time constants used in the multiexponential fit functions for these traces are listed in Table $\mathbf{S 3}$ below, which lists some of the same information as Table 2 in the main text. 
Table S3. Time Constants (on the Picosecond Timescale Only) for Excited-State Decay of CdS QDs with 1000 eq of added NB and 1000 eq of added 3-MPA, Monitored at Three Probe Wavelengths. ${ }^{a}$

\begin{tabular}{|l|l|l|l|l|l|l|l|}
\hline $\begin{array}{l}\text { CdS-NB- } \\
\text { MPA }\end{array}$ & $\tau_{1}\left(\mathrm{~A}_{1}\right)$ & $\tau_{2}\left(\mathrm{~A}_{2}\right)$ & $\tau_{3}\left(\mathrm{~A}_{3}\right)$ & & $\tau_{4}\left(\mathrm{~A}_{5}\right)$ & $\tau_{5}\left(\mathrm{~A}_{6}\right)$ \\
\hline $\mathrm{h}^{+}$ & $\begin{array}{l}\mathrm{e}^{-} \\
\text {trapping } \\
\text { or } \\
\text { cooling }\end{array}$ & $\begin{array}{l}\mathbf{h}^{+} \\
\text {transfer } \\
\text { to MPA }\end{array}$ & $\begin{array}{l}\mathrm{h}^{+} \\
\text {trapping }\end{array}$ & $\begin{array}{l}\mathrm{e}^{-} \\
\text {trapping }^{c}\end{array}$ & $\begin{array}{l}\mathrm{e}^{-} \\
\text {transfer } \\
\text { to NB }\end{array}$ & $\begin{array}{l}\text { radiative } \\
\mathrm{CR} \\
\left(\mathrm{e}^{-} \text {and }^{+}\right)\end{array}$ \\
\hline $\begin{array}{l}\mathbf{4 2 0} \mathbf{~ n m} \\
\left(\mathbf{e}^{-} \mathbf{~ o n l y )}\right.\end{array}$ & -- & $\begin{array}{l}1.6 \mathrm{ps} \\
(-0.08)\end{array}$ & -- & -- & $\begin{array}{l}227 \mathrm{ps} \\
(0.28)\end{array}$ & $\begin{array}{l}1.7 \mathrm{~ns} \\
(0.06)\end{array}$ & $\begin{array}{l}>3 \mathrm{~ns} \\
(0.74)\end{array}$ \\
\hline $\begin{array}{l}\mathbf{1 2 5 0} \mathbf{~ n m} \\
(\mathbf{m o s t l y} \\
\left.\mathbf{h}^{+}\right)\end{array}$ & $\begin{array}{l}0.44 \mathrm{ps} \\
(0.61)\end{array}$ & -- & $\begin{array}{l}5.7 \mathrm{ps} \\
(0.15)\end{array}$ & $\begin{array}{l}63 \mathrm{ps} \\
(0.10)\end{array}$ & -- & $\begin{array}{l}1.7 \mathrm{~ns} \\
(0.11)\end{array}$ & $\begin{array}{l}>3 \mathrm{~ns} \\
(0.03)\end{array}$ \\
\hline $\begin{array}{l}\mathbf{9 5 0} \mathbf{~ n m} \\
\left(\mathbf{e}^{-} \mathbf{a n d ~ h} \mathbf{h}^{+}\right)\end{array}$ & $\begin{array}{l}0.44 \mathrm{ps} \\
(0.25)\end{array}$ & -- & $\begin{array}{l}5.7 \mathrm{ps} \\
(0.17)\end{array}$ & $\begin{array}{l}63 \mathrm{ps} \\
(0.09)\end{array}$ & $\begin{array}{l}227 \mathrm{ps} \\
(0.13)\end{array}$ & $\begin{array}{l}1.7 \mathrm{~ns} \\
(0.13)\end{array}$ & $\begin{array}{l}>3 \mathrm{~ns} \\
(0.24)\end{array}$ \\
\hline
\end{tabular}

${ }^{a}$ Each lifetime is the average of two measurements on separately prepared samples that differed by less than $50 \%$. The quantities in parentheses are the fractional amplitudes of each component at each probe wavelength: $\Sigma_{\mathrm{i}} \mathrm{A}_{\mathrm{i}}=1$.

${ }^{b}$ A growth component: delayed formation of the GS bleach due to excitation of the sample at an energy greater than the bandgap of the QDs. Typically not seen in NIR TA spectra, and not relevant to the catalysis, so not discussed in the main text.

${ }^{c}$ An ultrafast electron trapping process not relevant to the catalysis and therefore not discussed in the main text.

\section{References}

(1) Knowles, K. E.; Tagliazucchi, M.; Malicki, M.; Swenson, N. K.; Weiss, E. A. J. Phys. Chem. C 2013, 117, 15849 . 